Journal of Computer Science 7 (11): 1685-1690, 2011

ISSN 1549-3636

(C) 2011 Science Publications

\title{
A Challenge for Routing Algorithms in Optical Multistage Interconnection Networks
}

\author{
Mehrnaz Moudi and Mohamed Othman \\ Department of Communication Technology and Network, \\ Faculty of Computer Science and Information Technology, \\ University Putra Malaysia, 43400 UPM, Serdang, Selangor D.E., Malaysia
}

\begin{abstract}
Problem statement: A class of dynamic interconnection networks is Multistage Interconnection Networks (MINs) that connects input devices to output devices through a number of switch stages. MINs have assumed importance in recent years; because of their cost-effectiveness. Optical MINs are one type of MINs that have large transmission capacity in the communication networks. There is a major problem in Optical MIN that is crosstalk, which is caused by coupling two signals within a switching element. Approach: To avoid crosstalk in Optical MINs many algorithms have been proposed by many researchers that we review applying five routing algorithms and scheduling them in the Optical MINs. Results: The comparative results of routing algorithms show affective of avoiding crosstalk in number of passes and execution time for different algorithm. Conclusion: The challenge between these routing algorithms is thoroughly investigated, by applying them on Optical MIN and showing which algorithm has better performance to avoid crosstalk.
\end{abstract}

Key words: Optical multistage interconnection networks, routing algorithm, switching element, Multistage Interconnection Networks (MINs), Switching Elements (SEs), Multilayer Multistage Interconnection Networks (MLMINs), Simulated Annealing (SA), Genetic Algorithm (GA)

\section{INTRODUCTION}

The limitation of most digital systems' performance is by their communication or interconnection (Katangur et al., 2004). The interconnection network implements a main role in defining the overall performance of a multicomputer system. If the network cannot provide sufficient performance for particular application, messages will frequently be required to wait for data to arrive. Some of these applications are telephone switches, interconnection networks for multicomputer and distributed shared memory multiprocessors, clusters of workstations and personal computers, local area networks, wide area networks and networks in industrial applications (Denko et al., 2009).

Nowadays, Multistage Interconnection Networks (MINs) have importance because of their costeffectiveness and is very popular in switching and communication applications. MINs are used in multiprocessing systems to provide cost-effective, highbandwidth communication between processors and/or memory modules and in used in telecommunication and parallel computing systems for many years. A MIN normally connects $\mathrm{N}$ inputs (sources) to $\mathrm{N}$ outputs (destinations) and is referred to as an $\mathrm{N} \times \mathrm{N}$ MIN. The parameter $\mathrm{N}$ is called the size of the network. It has $\mathrm{n}$ stages $\left(\mathrm{n}=\log _{2} \mathrm{~N}\right)$. Each stage has N/2 Switching Elements (SEs); each SE has two inputs and two outputs connected in a certain pattern. MINs can be electronic MINs or optical MINs that in electronic MINs electricity are used, where as in optical MINs light is used to transmit the messages (Aggarwal and Kaur, 2008).

Optical MINs (OMINs) are an attractive solution in the design of high-speed communication networks and switches because of having high bandwidth, low error probability and large transmission capacity. The electronic MINs and the optical MINs have many similarities, but crosstalk problem in the optical switches is one major difference between them (Sharma et al., 2008). Recently, a new architecture is introduced that applies especially to multicasting in multistage interconnection networks. In addition to multicast traffic, Tutsch and Brenner (2003) provide additional

Corresponding Author: Mehrnaz Moudi, Department of Communication Technology and Network,

Faculty of Computer Science and Information Technology, University Putra Malaysia,

43400 UPM, Serdang, Selangor D.E., Malaysia 
views on MINs performance aspects under special cases using simulations. Those MINs are called Multilayer Multistage Interconnection Networks (MLMINs) that their needs are for routing capacity in the presence of multicast and broadcast traffic, their performance prediction and evaluation but in this study we focus on optical multistage interconnection networks (Vasiliadis et al., 2009; 2010).

The study is organized as follows. Second section will give an overview about the major problem in Optical Multistage Interconnection Networks. Third section explains about the window method and general description of the methodology. In fourth section we have reviewed five algorithms that are usual to use in this case. Fifth section is related to compare algorithms and final section gives the conclusion.

Problem overview: Crosstalk in OMINs is an important problem. To avoid crosstalk problem which is caused by coupling two signals within a switching element (Almazyad, 2011), various approaches have been proposed by many researchers. In this research, we are interested in a network called optical omega network which has shuffle-exchange connection pattern. to send messages from a source to a destination on Optical Omega Network without crosstalk, we need to divide the messages into several groups. We want to separate the messages without conflicts with other messages in the same group as well as we want to reduce the total number of the groups. Under the constraint of avoiding crosstalk, three approaches, space domain, time domain and wavelength domain have been proposed.

In this research, crosstalk-free scheduling algorithms are reviewed based on the time domain approach to schedule messages for routing more efficiently in the Optical Omega Networks and the goal is to review efficient algorithm to send the messages in less time. There are some algorithms that have been proposed to avoid crosstalk in routing messages through an $\mathrm{N} \times \mathrm{N}$ optical network by many researchers.

\section{MATERIALS AND METHODS}

Window Method is description of a method that used to find conflict between input and output pair in electronic MINs and OMINs. The goal of routing algorithms is to minimize the number of passes to route all the inputs to the outputs with minimal crosstalk.
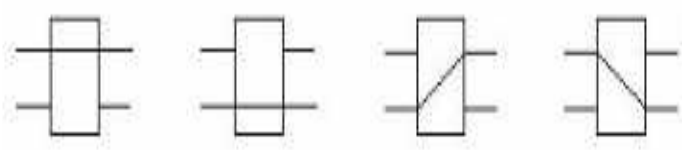

Fig. 1: Passing ways in a switch element
There is a rest that the crosstalk can be avoided by employing the pattern shown in Fig. 1. As sending messages to the network are in several groups, Window Method is used to find out which messages should not be in the same group because of crosstalk. At first combined each source address and its corresponding destination address to produce a combination matrix, it use a window size of $\mathrm{m}$, where $\mathrm{m}=\log _{2} \mathrm{~N}$ and $\mathrm{N}$ is the size of the network to determine the conflicts on the produced matrix (Abed and Othman, 2007; Bashirov and Karanfiller, 2010). The conflict matrix is a square matrix with $\mathrm{N} \times \mathrm{N}$ entry that $\mathrm{N}$ is the size of the network. With use of the window method's output, the Conflict Matrix $\left(\mathrm{M}_{\mathrm{ij}}\right)$ can be shown by assigning the value 1 for having a conflict between the source and destination and the value 0 is assigned if there is not a conflict (Abed and Othman, 2007). Then, based on the conflict matrix, the messages are sorted and selected for scheduling that different routing algorithms will be investigated in the next section.

Routing algorithms: We briefly review routing algorithms that purpose of these routing algorithms is to schedule the messages in different independent subsets in order to avoid the path conflicts in the network (Shanmugam et al., 2011; Potti and Chinnasamy, 2011). Five algorithms of routing algorithms that solve the crosstalk problem in MIN are four heuristic algorithms, Genetic Algorithm (GA), Simulated Annealing algorithm (SA), Zero algorithm and Ant Colony Optimization algorithm (ACO).

Four heuristic algorithms: There are many ways to decide the order of the scheduling. The four heuristic algorithms choose the message in the following way:

- The sequential increasing: Choose a message sequentially in increasing order of the message source address

- The sequential decreasing: Choose a message sequentially in decreasing order of the message source address

- Degree increasing: Choose a message based on the order of increasing degrees in the conflict graph

- Degree descending: Choose a message based on the order of decreasing degrees in the conflict graph

The degree of each message in the conflict graph is the number of conflicts to other messages it has in the conflict graph. Based on (Al-Shabi and Othman, 2008), scheduling the messages in decreasing degrees of the 
message conflicts will result in the best performance among these four algorithms.

Genetic algorithm: Genetic Algorithms (GAs) are one of routing algorithms. A GA is started with a set of represented solutions by chromosomes. Those solutions are called the population. Solutions from the initial population are taken and used to form a new population. This procedure is forced by the hope that the new population will be better than the old one. To select new solutions to form new population, the strength of the original solution is the important standard. If the fitness value is more, the more chances they get to reproduce. Generation by generation is repeated until some condition is satisfied such as the number of populations gets to the limit or the improvement of the best solution is good enough for the research or there is no more improvement possible (Othman and Shahida, 2010). The Genetic algorithm produces better results for number of passes in compare with the four heuristic algorithms but it was time consuming.

Simulated Annealing algorithm: Simulated Annealing originated in the annealing processes found in thermodynamics and metallurgy. Simulated Annealing is used to approximate the solution of very large combinatorial optimization problems (e.g. NP-hard problems). It is based upon the analogy between the annealing of solids and solving optimization problems. First time that SA was proposed, it was mostly known for its effectiveness in finding near optimal solutions for large-scale combinatorial optimization problems, such as the traveling salesperson problem, buffer allocation in production lines and chip placement problems in circuits (Abdullah et al., 2008).

The most important thing about simulated annealing is analogous to what happens when metals are cooled at a controlled rate. The slowly falling temperature allows the atoms in the molten metal to line them up and form a regular crystalline structure that has high density and low energy. But if the temperature goes down too quickly, the atoms do not have time to orient themselves into a regular structure and the result is a more amorphous metal with higher energy. In simulated annealing, the value of an objective function that wants to minimize is analogous to the energy in a thermodynamic system.

The basic Simulated Annealing algorithm includes the following steps (Abdullah et al., 2008):

- Start: Select a large starting value for the temperature $\mathrm{T}$, a value for the final temperature Tfinal, a value for the stopping condition ts that is chosen as 10 , correct value for the temperature reducing parameter. The ts value is used because it will stop the algorithm if there are no changes to the solution after ts iterations. We also choose a variable $M$ so that $M$ iterations are performed for each temperature

- Initialize the current gain to be equal to the gain of the solution, which we are using to precede and ts to some variable tstop

- While (tstop $>0$ and T $>$ Tfinal) we proceed

- $\quad$ For i $=1$ to $\mathrm{M}$ do

- Generate a new solution using the present solution.

- Calculate the new gain .If the new gain is less than the current gain we accept it, otherwise we use the following strategy to decide:

$$
\begin{gathered}
\mathrm{R}=\operatorname{random} \text { number }(0<\mathrm{R}<1) \\
\mathrm{Y}=\exp (-\Delta \mathrm{E} / \mathrm{T})
\end{gathered}
$$

- If $(\mathrm{R}<\mathrm{Y})$ then we accept

- the solution, else we reject it

- If we accept the move then the current gain will be set to the new gain and we keep the new solution, otherwise we keep the old solution

- If we accept the solution then we assign tstop = ts else we decrement tstop by 1 (tstop $=$ tstop -1 )

- Then we change the temperature by a factor $\alpha, \mathrm{T}=$ $\mathrm{T} * \alpha$

- $\quad$ End

The Simulated Annealing algorithm in compare with four heuristic algorithms improves the average number of passes. When compared to the Genetic algorithm the Simulated Annealing algorithm performance is almost equal to that but for large network sizes the Genetic algorithm is better. The main advantage of SA algorithm over Genetic algorithm is the running time because of time consuming.

Zero algorithms: The Zero algorithms have three types of algorithms that named the ZeroX, ZeroY and ZeroXY algorithms. These algorithms are based on routing algorithms to minimize the number of passes to route all the inputs to outputs without crosstalk. In addition, these algorithms when implemented with partial ZeroX and ZeroY algorithms would yield the same results as the other heuristic algorithms, but over performing them when the execution time is considered (Al-Shabi and Othman, 2008).

The technique in both ZeroX and ZeroY algorithms is based on grouping of messages that have summation equals to zero in the conflict matrix. The steps in both ZeroX and ZeroY algorithms are the same except in the summation process. ZeroX algorithm works by computing the sum of all entries in each 
column of the conflict matrix, while ZeroY algorithm computes for each row of the matrix. ZeroXY algorithm is a combination of both ZeroX and ZeroY algorithms. The result from ZeroXY algorithm is better than the result of ZeroX and ZeroY algorithms. Based on the experimental results shown Al-Shabi and Othman (2008), Zero algorithms perform best in terms of the execution and result very closely to the Degree Descending algorithm in terms of the number of passes generated to route a permutation. However, it was found Abed and Othman (2007) that crosstalk may still occur between messages scheduled in the same pass of a particular time slot using the original Zero algorithms.

Ant colony optimization algorithm: In the early 90's, Ant Colony Optimization (ACO) was introduced by Marco Dorigo and his co-workers. The ant colony algorithm is a technique to find optimal paths. It is based on the behavior of ants can always find the shortest path from a food source to their colony without visual cues. When reaching a point for decision, the ants will follow the trail that has more pheromone deposits. The ant colony algorithm can be used to the routing problem. An ACO algorithm designed to help solve the routing problem in telecommunications networks because the ant-colony works on a very dynamic system such as systems include computer networks and artificial intelligence simulations of workers. Some researcher use ACO algorithm for base of their researches in different purposes such as Parallel Ant Colony Optimization Algorithm, Solution to the problem of ant being stuck by ant colony routing algorithm (Zhao and Tong, 2009) and but for this study, the purpose is to review message routing and scheduling in Optical Multistage Networks by Ant Colony Optimization algorithm.

To route the messages in different passes such that they do not have any link conflicts (electrical crosstalk) between them but can have switch conflicts satisfying the ' $\mathrm{C}$ ' limited crosstalk constraint for an $\mathrm{N} \times \mathrm{N}$ optical network. The conflict graph is a graph $\mathrm{G}=(\mathrm{V}, \mathrm{E})$ where $\mathrm{V}$ and $\mathrm{E}$ are set of vertices and edges. We can assign a different color to each pass or group in which messages are routed. So we can transform our problem to that of a graph-coloring problem but in which adjacent nodes can have the same color if there are no link conflicts between them and if the node does not violate the ' $\mathrm{C}$ ' limited crosstalk. The graph coloring algorithm ANTCOL designed by Costa and Hertz using ant colony optimization techniques can successfully color graphs. In this algorithm, a given number of ants change the color of each node that they visited according to a local criterion when they move around the nodes of the graph. Ant moves from the current node to the adjacent node according to the maximum number of violations and the trail intensity. For minimizing the number of violations, the ant replaces the old color of the node with a new color that. This action is randomly repeated for each ant. The ant colony optimization technique improves the performance of routing and scheduling in OMIN when limited crosstalk is allowed and reduced the number of passes (Dorigo et al., 2011; Katangur et al., 2004).

As ACO uses are for combinatorial optimization problems such as the communication network routing problem. There is Improved Ant Colony Optimization (IACO) technique that it adjusts a new strategy, called the ant-weight strategy, to update the increased pheromone and a mutation operation, to solve the problem. You can see this algorithm in (Zhaoa et al., 2010).

\section{RESULTS}

In relation to previous researches, in Four Heuristic Algorithm scheduling the messages in decreasing degrees of the message conflicts resulted in the best performance among four heuristic algorithms. According to the researcher's result, Ant Colony Optimization algorithm is better than other algorithms to decrease number of passes. The average execution times by using routing algorithms in different network sizes show that when the size of network increases, the execution time is different in all of the algorithms. The execution time in Genetic algorithm is more than other algorithms. Simulated Annealing algorithm has less execution time in compare with other algorithms. Finally, in compare with four previous algorithms, Zero algorithms perform better and minimize the number of passes and improve the execution time.

\section{DISCUSSION}

Different routing algorithms were compared in terms of the number of passes and execution time that previous researchers found. The following Table 1 shows the comparison of different routing algorithms, although some conditions were assumed for some routing algorithms to get results (Katangur et al., 2004). The main challenge for these routing algorithms is for avoiding crosstalk. The average number of passes by using routing algorithms in different network sizes show when the size of network increase, the results change and from this changing we can understand that which algorithm decreases more number of passes. 
Table 1: Comparison of five routing algorithms for solving the crosstalk in OMIN

\begin{tabular}{|c|c|c|}
\hline Algorithm & Scheduling Model & Performance \\
\hline $\begin{array}{l}\text { Four heuristic } \\
\text { (Al-Shabi and Othman, 2008; } \\
\text { Othman and Shahida, 2010) }\end{array}$ & $\begin{array}{l}\text { Sequential Increasing, } \\
\text { Sequential Decreasing, } \\
\text { Degree Increasing and } \\
\text { Degree Descending }\end{array}$ & $\begin{array}{l}\text { Decreasing degree has best performance to improves the } \\
\text { average number of passes and execution time }\end{array}$ \\
\hline $\begin{array}{l}\text { Genetic } \\
\text { (Othman and Shahida, 2010) }\end{array}$ & $\begin{array}{l}\text { Starting with a set of represented } \\
\text { solutions by chromosomes }\end{array}$ & $\begin{array}{l}\text { Generation by generation is repeated until some condition is } \\
\text { satisfied (the conditions depend to the kind of problem) } \\
\text { and it improves the average number of passes especially for } \\
\text { large network size but is time consuming }\end{array}$ \\
\hline $\begin{array}{l}\text { Simulated annealing } \\
\text { (Abdullah } \text { et al., 2008) }\end{array}$ & $\begin{array}{l}\text { The analogy between the annealing of solids } \\
\text { and solving optimization problems }\end{array}$ & $\begin{array}{l}\text { Mostly known for its effectiveness in finding near } \\
\text { optimal solutions for optimization problems and Improves } \\
\text { the average number of passes and execution time }\end{array}$ \\
\hline $\begin{array}{l}\text { Ant Colony Optimization } \\
\text { (Katangur } \text { et al., 2004) }\end{array}$ & $\begin{array}{l}\text { Based on the behavior of ants can always find } \\
\text { the shortest path from a food source to their } \\
\text { colony Assign a different color to each pass or } \\
\text { group in which messages are routed }\end{array}$ & $\begin{array}{l}\text { This technique improves the performance of routing and } \\
\text { scheduling in OMIN when limited crosstalk is allowed and } \\
\text { improves the average number of passes and execution time }\end{array}$ \\
\hline $\begin{array}{l}\text { Zero } \\
\text { (Al-Shabi and Othman, 2008) }\end{array}$ & $\begin{array}{l}\text { Grouping of messages that have summation equals } \\
\text { to zero in the conflict matrix }\end{array}$ & $\begin{array}{l}\text { Three types of algorithm: } \\
\text { ZeroX, ZeroY and ZeroXY } \\
\text { These algorithms are based on routing algorithms to } \\
\text { minimize the number of passes to route all the inputs } \\
\text { to outputs without crosstalk and it is better to improve the } \\
\text { average number of passes and execution time }\end{array}$ \\
\hline
\end{tabular}

\section{CONCLUSION}

Throughout this study, we have presented the crosstalk-free scheduling algorithms for routing in Optical MINs. Efficient message routing algorithms directly affect the performance of communication networks. The main advantage of our proposed routing algorithm is that it makes the Optical MINs crosstalk free. We use all above algorithms to improve the performance of routing and scheduling in optical multistage networks.

Based on the performance evaluated by previous researchers, the Simulated Annealing algorithm over the other Four Heuristic algorithms is that it improves the average number of passes. In compared with the Genetic algorithm the Simulated Annealing algorithm performance is almost equal with the Genetic algorithm and, for large network sizes, it is better than the Genetic algorithm because it decrease running time. Zero algorithms perform best in terms of the execution time and the number of passes generated to route a permutation. On the other hand, the ACO algorithm can successfully reduce the number of passes for routing the messages when limited crosstalk is allowed. The ant algorithm improves the solution because ants act as cooperative agents in parallel for finding a solution.

\section{ACKNOWLEDGEMENT}

The study described in this study was supported by the Research University Grant Scheme (RUGS),
University Putra Malaysia (RUGS Number: 05/01/07/0180RU).

\section{REFERANCES}

Abdullah, M., M. Othman, H. Ibrahim, \& S. Subramaniam. 2008. Simulated annealing algorithm for scheduling Divisible Load in large scale data grids. Proceedings of the International Conference on Computer and Communication Engineering, May 13-15, IEEE Xplore Press, Kuala Lumpur, pp: 1032-1036. DOI: 10.1109/ICCCE.2008.4580765

Abed, F. and M. Othman, 2007. Efficient window method in optical multistage interconnection networks. Proceedings of the IEEE International Conference on Telecommunications and Malaysia International Conference on Communications, May 14-17, IEEE Xplore Press, Penang, pp: 181-185. DOI: 10.1109/ICTMICC.2007.4448626

Aggarwal, R. and L. Kaur, 2008. Design and bandwidth analysis of fault-tolerant multistage interconnection networks. J. Comput. Sci., 4: 963-966. DOI: 10.3844/jcssp.2008.963.966

Almazyad, A.S., 2011. Optical omega networks with centralized buffering and wavelength conversion. J. King Saud Univ. Comput. Inform. Sci., 23: 15-28. DOI: $10.1016 /$ j.jksuci.2010.03.002 
Al-Shabi, M. and M. Othman, 2008. A new algorithm for routing and scheduling in optical omega network. Int. J. Comput. Internet Manage., 16: 2631.

Bashirov, R. and T. Karanfiller, 2010. On path dependent loss and switch crosstalk reduction in optical networks. Inform. Sci., 180: 1040-1050. DOI: 10.1016/J.INS.2009.11.017

Denko, M.K., L.T. Yang and Y. Zhang, 2009. Autonomic Computing and Networking. 1st Edn., Springer, New York, ISBN-10: 0387898271, pp: 464.

Dorigo, M., G.D. Caro and L.M. Gambardella, 2011. Ant algorithms for discrete optimization. Art. Life, 5: 137-172. DOI: 10.1162/106454699568728

Katangur, A.K., S. Akkaladevi, Y. Pan and M.D. Fraser, 2004. Applying ant colony optimization to routing in optical multistage interconnection networks with limited crosstalk. Proceedings of the 18th International Presented at the Parallel and Distributed Processing Symposium, Apr. 26-30, IEEE Computer Society, Santa Fe, New Mexico, pp: 163-163. DOI: 10.1109/IPDPS .2004.1303156

Othman, M. and T.D. Shahida, 2010. The development of crosstalk-free scheduling algorithms for routing in optical multistage interconnection networks. Trends Telecommun. Technol.

Potti, S. and C. Chinnasamy, 2011. Strength pareto evolutionary algorithm based multi-objective optimization for shortest path routing problem in computer networks. J. Comput. Sci., 7: 17-26. DOI: $10.3844 / j \operatorname{cssp} .2011 .17 .26$
Shanmugam, G., P. Ganesan and P.T. Vanathi, 2011. Meta heuristic algorithms for vehicle routing problem with stochastic demands. J. Comput. Sci., 7: 533-542. DOI: 10.3844/jcssp.2011.533.542

Sharma, S., P.K. Bansal and K.S. Kahlon, 2008. On a class of multistage interconnection network in parallel processing. Int. J. Comput. Sci. Network Sec., 8: 287-291.

Tutsch, D. and M. Brenner, 2003. MINSimulate-a multistage interconnection network simulator.

Vasiliadis, D., G. Rizos and C. Vassilakis, 2010. Performance study of multilayered multistage interconnection networks under hotspot traffic conditions. J. Comput. Syst. Networks Commun., 1-11. DOI: $10.1155 / 2010 / 403056$

Vasiliadis, D.C., G.E. Rizos, C. Vassilakis and E. Glavas, 2009. Performance evaluation of multicast routing over multilayer multistage interconnection networks. Proceedings of the 5th Advanced International Conference on Telecommunications, May 24-28, IEEE Computer Society, Venice/Mestre, Italy, pp: 395-403. DOI: 10.1109/AICT.2009.72

Zhao, J. and W.M. Tong, 2009. Solution to the problem of ant being stuck by ant colony routing algorithm. J. China Univ. Posts Telecommun., 16: 100-105. DOI: $10.1016 / \mathrm{S} 1005-8885(08) 60187-9$

Zhaoa, D., L. Luo and K. Zhang, 2010. An improved ant colony optimization for the communication network routing problem. Math. Comput. Modell., 52: 1976-1981. DOI:10.1016/j.mcm.2010.04.021 